\section{Another new tool for the diagnostic bronchoscopist}

\author{
Robert C Rintoul, Mark G Slade
}

Solitary pulmonary nodules (SPNs), defined as rounded lesions $<3 \mathrm{~cm}$ completely surrounded by an aerated lung, are increasingly identified by modern chest radiography and thoracic CT. As CT screening studies report and the debate over the place of national lung cancer screening programmes intensifies, the need for algorithms to efficiently investigate SPNs will become even more important. ${ }^{1}$

Peripheral pulmonary lesions can be biopsied by various techniques. The most commonly used approach in the UK is transthoracic needle aspiration or core biopsy usually performed under CT guidance. The diagnostic sensitivity for malignancy exceeds $90 \%$ in experienced hands but up to $44 \%$ of biopsies in benign disease are non-diagnostic. ${ }^{2}$ While generally safe, it is not without complications; in a large UK-based survey, pneumothorax occurred in $20.5 \%$ of cases and intercostal tube drainage was required in $3 \% .^{3}$

Flexible bronchoscopes can be used to approach peripheral lesions but the reported diagnostic sensitivity rates are considerably lower than for CT-guided needle biopsy. Sensitivity has been shown to be dependent upon the size of lesion, the distance from the hilum and the presence of a bronchus leading to the lesion on CT (the CT bronchus sign). A systematic review found that the diagnostic yield was $33 \%$ for lesions $<2 \mathrm{~cm}$ in the outer third of the lung. ${ }^{2}$ Historically, the approach taken has been to use a thin bronchoscope and perform a combination of bronchial washings, brushings and transbronchial biopsy targeting the appropriate segmental or subsegmental bronchus sometimes with the assistance of fluoroscopy. More recently, several groups have reported their experience of using radial endobronchial ultrasound (EBUS) to guide biopsy of peripheral lesions. Radial EBUS provide $360^{\circ}$ images of the airway wall and surrounding structures. Previously, this technique was

Department of Thoracic Oncology, Papworth Hospital, Cambridge, UK

Correspondence to Dr Robert C Rintoul, Department of Thoracic Oncology, Papworth Hospital, Cambridge CB23 3RE, UK; robert.rintoul@nhs.net often employed to identify the position of parabronchial or paratracheal lymph nodes prior to sequential (non-ultrasound guided) transbronchial needle aspiration (TBNA). ${ }^{4}$ However, this application has essentially been superseded by the development of linear EBUS. Nowadays, radial EBUS is predominantly used to localise a peripheral lesion and allow placement of a guide sheath through which biopsies can be taken. Diagnostic yields in the range of $58-80 \%$ have been reported. ${ }^{5-9}$ While radial EBUS helps with localisation of a peripheral lesion, the problem of navigating to the appropriate bronchus remains, which results in a long learning curve and lengthy procedures and has in large part been responsible for the low uptake of the technique in clinical practice. Therefore, in recent years considerable effort has been directed at the development of navigation systems to aid bronchoscope placement. ${ }^{10-12}$ Eberhardt et al showed that a combination of electromagnetic navigation and radial EBUS was superior to either technique alone. In Thorax, Ishida et al report a randomised trial that assessed the role of a virtual bronchoscopic navigation system to facilitate radial EBUS placement of a guide sheath prior to biopsy of peripheral pulmonary lesions. ${ }^{13}$ The diagnostic yield using the navigation system in combination with EBUS was $80 \%$, significantly higher than in the control arm (67\%), which used EBUS alone. The duration of the examination was shorter (24.0 vs $26.2 \mathrm{~min}, \mathrm{p}=0.016$ ), which is an important consideration in a procedure performed under sedation. The navigation system employed sophisticated computer reconstruction of virtual bronchoscopic images alongside standard video bronchoscopy. Although not widely available at this time, the approach used by Ishida and colleagues appears simpler and easier to use than some of the other commercially available navigation systems. However, at this time no head-to-head studies of the various navigation system approaches have been published.

The improvements in the diagnostic yield of bronchoscopic approaches increase the options available to clinicians investi- gating peripheral lesions. Increasingly, respiratory physicians are growing in confidence and experience with ultrasound. In the same way that linear EBUS-TBNA has been taken up by many bronchoscopists who previously lacked confidence to perform conventional TBNA, it is likely that improved navigation systems will encourage the spread of radial EBUS for biopsy of peripheral lesions. At present, however, experience in radial EBUS is limited to a relatively small number of specialist centres.

Faced with a choice of biopsy techniques, the question arises as to how best to approach a SPN. In considering this, a number of factors need to be taken into account. Clearly, accessibility to a technique and local expertise will play a significant role. At present, in the majority of centres percutaneous biopsy is more readily available and has a high sensitivity for malignancy. However, the risk of pneumothorax with transthoracic biopsy is considerably higher than that reported for endobronchial approaches ${ }^{5-7}$ 9 and for patients in whom pneumothorax may cause significant clinical compromise, navigation-aided radial EBUS may be an appropriate first approach especially if there is CT evidence of an air bronchogram sign-an airway leading directly to the lesion. $^{11}$

In assessing a patient with suspected lung cancer, it is essential to consider the extent of disease and target biopsies appropriately. Although the updated NICE guidelines for the management of lung cancer (2011) omitted radial EBUS for lack of available evidence, one of the new recommendations is that clinicians should choose investigations that give the most information about diagnosis and staging with least risk to the patient. ${ }^{14}$ Not infrequently, patients undergo more than one biopsy procedure, first for diagnosis and then for staging, which are both arduous for the patient and costly to the NHS. For instance, in the past a patient may initially have had a transthoracic biopsy of a peripheral lesion before moving on to have mediastinal lymph node assessment by linear EBUS or mediastinoscopy. The new staging algorithms recommend that patients who may have disease suitable for treatment with curative intent should have enlarged mediastinal or other lesions biopsied in preference to the primary lesion. Identification of appropriate targets will come from the increasing use of PET-CT at an earlier time point in the staging algorithm to help direct biopsies. In the future, 
selected patients could have a single endobronchial procedure that comprises navigation-aided radial EBUS to biopsy a peripheral lesion combined with linear endobronchial and/or endoscopic ultrasound for mediastinal staging. Such an approach is already being undertaken, usually under general anaesthesia for patient comfort and tolerance, in a few highly specialised centres in continental Europe and the USA. However, further trials comparing the efficacy of endobronchial versus percutaneous biopsy strategies are required before firmer recommendations can be made.

As the diagnostic and staging algorithms for lung cancer evolve, it is essential that in parallel with developments in technology work is undertaken on quality of life and health resource use, an area that is frequently overlooked. The endobronchial approach to peripheral lesions is no exception and the approach documented by Ishida et $a l^{13}$ joins the existing literature on electromagnetic navigation systems, ultrathin bronchoscopy and fluoroscopyassisted bronchoscopy. Unfortunately, the current paper stopped short of incorporating an economic analysis, which was an opportunity missed.

The marked upturn in interest in thoracic oncology among respiratory physicians in recent years is partly stimulated by the growth in tools available to the bronchoscopist both for diagnosis and for therapy. Linear EBUS is gaining widespread acceptance and a number of centres are combining this procedure with endoscopic ultrasound. As navigation systems improve, it is likely that the use of radial
EBUS will also spread. While continued equipment development is to be encouraged, it will be equally important to address training and ensure that operators achieve and maintain high levels of diagnostic accuracy. This may require regional centralisation of specialist services in order to be cost and manpower effective. At present, other than recommendations that cancer networks in England and Wales should have access to these specialist techniques, ${ }^{14}$ there is no strategic development plan with the result that interested clinicians and trusts are developing services on an ad hoc basis. In addition to training, the challenge over the next few years will be to ensure that there is a coordinated approach to the development of specialist bronchoscopy services to allow equity of access for the population as a whole.

Competing interests Papworth Hospital is in receipt of loan equipment from Olympus UK, which provides a non-entailed educational grant to support the Papworth EBUS course of which RCR is the director.

Provenance and peer review Commissioned; internally peer reviewed.

Published Online First 7 October 2011

Thorax 2011:66:1027-1028.

doi:10.1136/thoraxjnl-2011-200921

\section{REFERENCES}

1. The National Lung Screening Trial Research Team. Reduced lung-cancer mortality with low-dose computed tomographic screening. N Engl J Med 2011;365:395-409.

2. Rivera MP, Mehta AC. Initial diagnosis of lung cancer: ACCP evidence-based clinical practice guidelines (2nd edition). Chest 2007:132:131S-48S

3. Richardson CM, Pointon KS, Manhire AR, et al. Percutaneous Lung biopsies: a survey of UK practice based on 5444 biopsies. Br J Radiol 2002; 75:731-5.

4. Herth FJ, Becker HD, Ernst A. Ultrasound-guided transbronchial needle aspiration: an experience in 242 patients. Chest 2003;123:604-7.

5. Paone G, Nicastri E, Lucantoni G, et al. Endobronchial ultrasound-driven biopsy in the diagnosis of peripheral lung lesions. Chest 2005;128:3551-7.

6. Herth FJ, Ernst A, Becker HD. Endobronchial ultrasound-guided transbronchial lung biopsy in solitary pulmonary nodules and peripheral lesions. Eur Respir J 2002;20:972-4.

7. Kurimoto N, Miyazawa T, Okimasa $S$, et al. Endobronchial ultrasonography using a guide sheath increases the ability to diagnose peripheral pulmonary lesions endoscopically. Chest 2004:126:959-65.

8. Chao TY, Chien MT, Lie CH, et al. Endobronchial ultrasonography-guided transbronchial needle aspiration increases the diagnostic yield of peripheral pulmonary lesions: a randomized trial. Chest 2009;136:229-36.

9. Fielding DI, Robinson PJ, Kurimoto N. Biopsy site selection for endobronchial ultrasound guide-sheath transbronchial biopsy of peripheral lung lesions. Intern Med J 2008:38:77-84.

10. Eberhardt R, Anantham D, Ernst A, et al. Multimodality bronchoscopic diagnosis of peripheral lung lesions: a randomized controlled trial. Am J Respir Crit Care Med 2007;176 36-41.

11. Seijo LM, de Torres JP, Lozano MD, et al. Diagnostic yield of electromagnetic navigation bronchoscopy is highly dependent on the presence of a bronchus sign on CT imaging: results from a prospective study. Chest 2010;138:1316-21.

12. Gildea TR, Mazzone PJ, Karnak, et al. Electromagnetic navigation diagnostic bronchoscopy: a prospective study. Am J Respir Crit Care Med 2006;174:982-9.

13. Ishida T, Asano F, Yamazaki K, et al. Virtual bronchoscopic navigation combined with endobronchial ultrasound to diagnose small peripheral pulmonary lesions: a randomised trial. Thorax 2011;66:1072-7.

14. National Institute for Health and Clinical Excellence. The diagnosis and treatment of lung cancer (update). 2011. http://guidance.nice.org.uk/ CG121/Guidance/pdf/English.

\section{Thorax alerts}

Sign up for our electronic table of contents alerts and you will never miss new issues of Thorax when published online. Stay ahead and up to date by visiting thorax.bmj.com. 